\title{
DESIGN AND OPERATION OF A PORTABLE, DIGITAL IMPULSE RADAR
}

\author{
By F.H.M. JonEs, B.B. NAROD, and G.K.C. Clarke \\ (Department of Geophysics and Astronomy, University of British Columbia, \\ Vancouver, British Columbia V6T 1W5, Canada)
}

\begin{abstract}
We have constructed a back-portable, impulse radar instrument that weighs roughly $12 \mathrm{~kg}$, can be powered by dry cells, and records and stores data digitally. The radar is controlled by an on-board, purpose-built microcomputer that ensures simple field operation, facilitates modifications, and allows unattended operation when investigating time-varying targets. The transmitting unit is triggered from the receiver via an optical link and generates an electromagnetic wavelet by discharging two high-voltage capacitors through back-to-back silicon-controlled rectifiers into a resistively loaded dipole antenna. Successful field operations carried out during the summers of 1986 and 1987 include depth profiling, as well as studies of englacial and subglacial targets, on several temperate and sub-polar glaciers.
\end{abstract}

\section{INTRODUCTION}

Cook (1960) first proposed the glaciological use of impulse radars and by the late 1970 s their value had become well established. For impulse sounding of glaciers, signals are commonly in the $1-20 \mathrm{MHz}$ range, and data are observed optically or recorded photographically (Sverisson and others, 1980; Watts and Wright, 1981) or as analogue signals on magnetic tape (Jacobel and Raymond, 1984; Brown and others, 1986). The scientific potential of digitized data has been demonstrated (Walford and others, 1986; Jacobel and Anderson, 1987) but most digital instruments in present use involve adaptations of laboratory equipment. Such equipment is cumbersome owing to requirements for environmental protection and suitable power sources. These factors led to our decision to develop a portable digital radar system. In this paper we describe the design and field testing of a prototype instrument.

Under ideal conditions, our system is capable of generating signals of approximately $4.5 \mathrm{~kW}$ instantaneous peak power, and detecting echoes to a depth of greater than $700 \mathrm{~m}$. Range accuracy depends both on instrumentation and glaciological factors, but only the former are considered here. Our effective sampling rate yields an instrumentation precision of better than $1 \mathrm{~m}$. The system is small, light (as little as $12 \mathrm{~kg}$ depending upon battery needs) and has low power consumption (11 W typical). Stability with respect to battery condition is ensured by using voltage regulators for all supply lines. A crystal-derived $1 \mathrm{MHz}$ signal is included for field verification of time-base accuracy. With the exception of transmitter drift discussed below, no instabilities with respect to temperature were found. Echograms are collected and stored digitally under user or program control. The latter feature enables unattended operation so that temporal variations can easily be monitored. The system is organized as two separate units: a transmitter module and a receiver module (Figs 1 and 2). The transmitter unit consists of an antenna and a pulse generator which together generate an impulse having a band width of up to $20 \mathrm{MHz}$ (the selection of the antenna determining the centre frequency). The receiver unit includes an identical antenna, a digitizing receiver, and a microcomputer of our own design. This microcomputer controls the acquisition of records and the operations of labelling, storing, retrieving, and displaying them. An optical cable links the two units, allowing the receiver unit to trigger the transmitter. Trace recording begins before the transmitter is triggered so that the complete surface wave is acquired and reliable timing is established.

A complete set of specifications appears in Table I. In the remainder of this paper we provide detailed descriptions of the transmitter and receiver units, discuss operational experience, and present representative data from our field seasons.

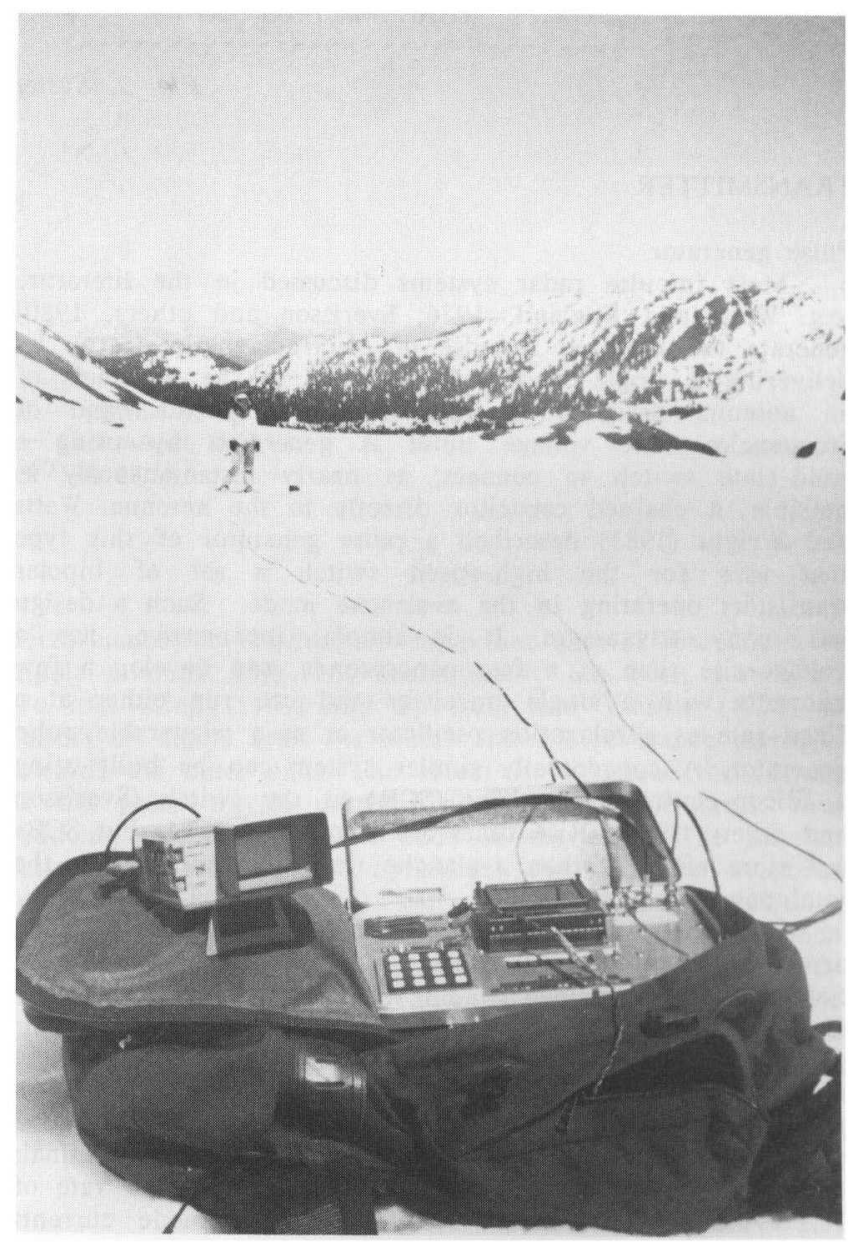

Fig. 1. Operating the U.B.C. impulse radar system from a back pack on Helm Glacier, British Columbia, Canada. Receiving unit and oscilloscope in the foreground are connected to the transmitter in the background by a fiber-optics cable. 


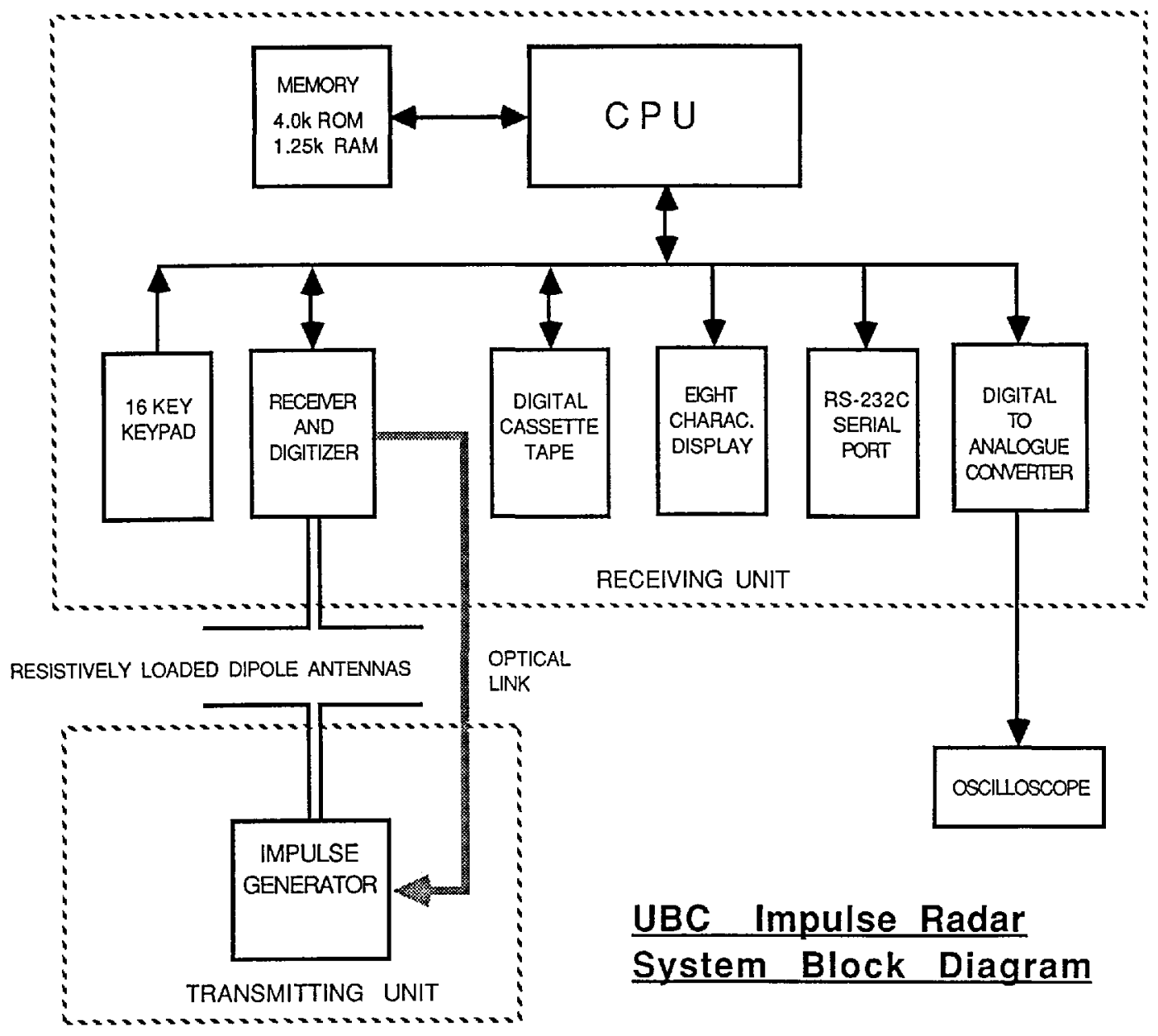

Fig. 2. System block diagram.

\section{TRANSMITTER}

\section{Pulse generator}

Most impulse radar systems discussed in the literature (e.g. Watts and England, 1976; Sverisson and others, 1980) generate the required impulse of electromagnetic energy by delivering a single-phase voltage pulse to the terminals of an antenna designed to radiate over a broad band of frequencies. The voltage pulse is generated by using a solid-state switch to connect, as nearly instantaneously as possible, a charged capacitor directly to the antenna. Watts and Wright (1981) described a pulse generator of this type that uses for the high-speed switch a set of bipolar transistors operating in the avalanche mode. Such a design has many advantages. It is simple, inexpensive, has a voltage-rise time of a few nanoseconds, can develop a few kilowatts with a single transistor and can run either at a fixed rate as a relaxation oscillator or as a triggerable pulse generator. A conceptually similar system can be built using a silicon-controlled rectifier (SCR) as the switch (Sverisson and others, 1980). Rise times are somewhat slower but SCRs are more efficient than avalanche transistors and reduce the total power requirements.

Our radar transmitter (Fig. 3) consists of two SCRs arranged back-to-back. Before switching, each one is holding off approximately $800 \mathrm{~V}$ at its anode. When they are simulataneously triggered by current pulses at their gates, the SCRs discharge a pair of capacitors into the antenna such that one arm of the dipole receives a $600 \mathrm{~V}$ positive pulse and the other arm receives a similar negative pulse. The resulting $1200 \mathrm{~V}$ pulse at the antenna terminals has an exponential rising edge which reaches a slew rate of $10.2 \mathrm{~V} / \mathrm{ns}$. As the anode voltages fall, the anode currents also fall until they drop below the so-called latching current. The SCRs then revert to their off conditions and the capacitors can recharge in time for the next trigger signal.

Common high-voltage SCRs (type $2 \mathrm{~N}-6399$ ) were used in the prototype transmitter. Although overall switching time is long, the avalanching process results in a fast slew rate. We have found that there is enough high-frequency energy contained in our switching wave form to drive $8 \mathrm{MHz}$ (center frequency) antennas (see, for example, the results shown in Figure 4). It is the early part of switching that is slow and temperature-sensitive, and this is where costly SCRs such as the $2 \mathrm{~N}-4204$ could be expected to improve transmitter performance.

Figure 3 is a schematic diagram of the power stage of the transmitter to which the following practical notes refer. (Complete specifications, circuit descriptions, and schematics can be found in Jones (unpublished).) Capacitors $C_{2}$ and $C_{3}$ were chosen to be high-voltage ceramic dise capacitors of $0.01 \mu \mathrm{F}$, and $\mathbf{R}_{2}$ and $\mathbf{R}_{3}$ limit the charging rate to suit the small d.c.-to-d.c. converter used as a high-voltage supply (Venus Scientific, Inc. model C8T; converts $12 \mathrm{~V}$ d.c. at $190 \mathrm{~mA}$ to $800 \mathrm{~V}$ d.c.). At the maximum charging rate, these capacitors take $4 \mathrm{~ms}$ to recharge, so the maximum pulse repetition rate is $250 \mathrm{~Hz}$. Resistors $R_{4}$ and $R_{7}$ are required to reference the antenna to ground and to provide a slow discharge path for the charge delivered to the antenna arms. These resistors must be large enough to minimize reverse antenna currents, preventing a secondary radiated pulse.

The trigger generator converts the optical trigger signal into a current pulse suitable for forcing the SCRs into an avalanching condition. A pulse transformer, $T_{1}$, with dual secondary windings ensures simultaneous timing of the two trigger currents, and resistors $R_{5}$ and $R_{6}$ are selected so that trigger currents are appropriate for the individual characteristics of each SCR. In this way, avalanche breakdown of both SCRs can be tuned to occur at precisely the same instant, as long as ambient conditions are identical, a condition ensured by placing the two devices adjacent to each other on the same heat sink.

During field trials we have found that overall timing is temperature-dependent, such that lower ambient temperatures result in greater delays between arrival of the trigger signal and emission of the radiated pulse. Delays of up to $3 \mu \mathrm{s}$ were observed at temperatures as low as $-15^{\circ} \mathrm{C}$, the lower 
System

Depth precision
Maximum depth

Data storage

Time to gather one record

Time to store one record

Automatic data collection

Weights (no batteries)
$0.82 \mathrm{~m}$

$841 \mathrm{~m}$

1320 bytes per record

52 records per cassette

$4 \mathrm{~s}$

$40 \mathrm{~s}$

Programmable -1 to $255 \mathrm{~min}$ intervals

Receiver unit - $5.1 \mathrm{~kg}$

Transmitter $-0.4 \mathrm{~kg}$

Antennas - $1.2 \mathrm{~kg}$ each

Transmitter

Peak pulse output

Pulse rise time

Pulse recovery

Repetition rate

Internal triggering delay

Power requirements

Antemas

Type

Dipole arm length

Centre frequency

Damping coefficient
$1200 \mathrm{~V}$ into antenna

40 ns from $37 \%$ to $100 \%$ of $V$

Time constant is approximately $30 \mu \mathrm{s}$

$250 \mathrm{~Hz}$

$0.8 u s$ at $24^{\circ} \mathrm{C}$

Idling $-95 \mathrm{~mA}$ at $18 \mathrm{~V}$

Pulsing at $250 \mathrm{~Hz}-280 \mathrm{~mA}$ at $18 \mathrm{~V}$

Construction. Wire and carbon resistors are installed inside $1 \mathrm{~m}$ sections of plastic pipe. Sections can be screwed together to form antennas with required centre frequency and damping characteristics.

Receiver unit

$\begin{array}{ll}\text { Input impedance } & 3.6 \mathrm{k} \Omega \\ \text { Total voltage gain } & 150 \\ \text { Attenuator } & \text { Divide by factors of 1, 2, 4, 20, or } 100 \\ \text { Sensitivity at maximum gain } & \mathrm{LSB}=0.26 \mathrm{mV} \text { at receiver antenna } \\ \text { Amplitude resolution } & 8 \mathrm{bits} \\ \text { Band width } & 46 \mathrm{MHz} \\ \text { Number of samples per record } & 1024 \\ \text { Sampling interval } & 9.76 \mathrm{~ns} \\ \text { CPU } & \text { RCA CDP1802 } \\ \text { Memory } & 4 \mathrm{kbyte} \text { of ROM } \\ \text { Clock speed } & 1.25 \mathrm{kbyte} \text { of RAM } \\ \text { Inputs and outputs } & 2.0 \mathrm{MHz} \\ & \text { Hex keypad } \\ & 8 \text { digit LED display } \\ & \text { Oscilloscope driver } \\ \text { PS-232C serial interface } \\ \text { Power requirements } & \text { Digital read/write cassette } \\ & \text { Min. (system idling) }-450 \mathrm{~mA} \text { at } 18 \mathrm{~V} \\ & \text { Max. (momentary) } 1800 \mathrm{~mA} \text { at } 18 \mathrm{~V} \\ & \text { Gathering data }-960 \mathrm{~mA} \text { at } 18 \mathrm{~V}\end{array}$

Note. All digital circuits use CMOS technology.

Broad-band (resistively damped) half-wave dipole

$h=5 \mathrm{~m}$

$8.4 \mathrm{MHz}$ in ice

$15 \mathrm{MHz}$ in air

$R_{0}=300 \Omega$ limit for reliable operation. This does not affect results because only the actual emission of the pulse is delayed. Since recording begins before the transmitter has fired, the time difference between receipt of the surface wave and receipt of the echo can be measured regardless of when the pulse is actually emitted. This is the chief advantage of synchronizing the transmitter to the receiver rather than the other way round.

The delay occurs because a greater charge (i.e. a longer current pulse) is required to initiate avalanching when semi-conductor junctions are at lower temperatures. This is not a fundamental problem. Use of higher-quality SCRs, temperature compensation, and more aggressive triggering would all contribute to more stable behaviour than that exhibited by our prototype.

This transmitter is not limited to use in triggered applications. For example, replacing the optical coupling section with a free-running oscillator would result in an efficient, self-contained impulse radar transmitter which could be used with a portable oscilloscope for a receiver in a manner similar to that described by Jezek and Thompson (1982).

Antennas

A broad-band antenna (one with good transient performance) is required to damp out natural resonances. A dipole antenna with this characteristic has been shown by Wu and King (1965) and by Rose and Vickers (1974) to consist of dipole arms for which internal resistance increases towards the outer ends. The effect is to reduce the current as the pulse travels outward along the dipole arms, thus reducing reflections at the ends of the arms. The internal resistance profile that optimizes the impulse response (regardless of efficiency) can be found using relations derived by $W u$ and King (1965), with corrections by Shen and King (1965). They showed that a pure outwardtravelling wave exists on an antenna arm of length $h$ if the internal impedance, $Z(x)$, at a position $x$ from the centre of the dipole, is given by

$$
Z(x)=\frac{\Phi \Gamma}{2 n(h-x)}=\frac{R_{0}}{h-x} .
$$

In Equation (1), the term $\Phi$ is the intrinsic impedance of the medium and $\Gamma$ is the ratio of vector potential on the antenna surface to current along the antenna at the point where the current is a maximum. For this optimally damped antenna, Shen and $K$ ing (1965) showed that the efficiency is around $9 \%$. $R_{0}$ should be reduced to find a compromise 


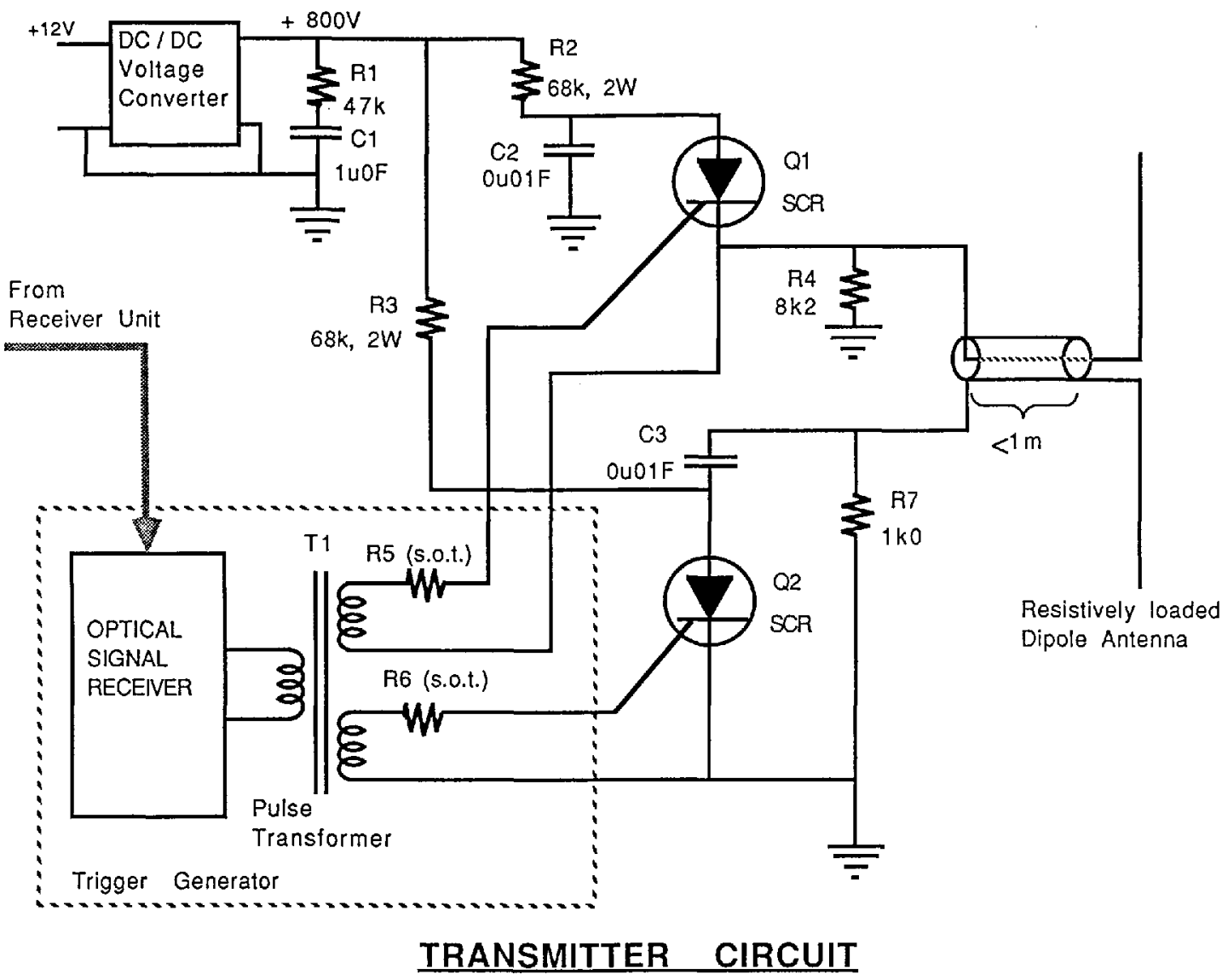

Fig. 3. Simplified circuit diagram of the transmitter referred to in the discussion of transmitter operation.

between sufficient damping and adequate radiated energy for sounding. In general, $R_{0}$ would not be the same for different antenna lengths, nor would a single $R_{0}$ be optimum for a given antenna on different glaciers.

For our initial work, the approximate relationships given by Wu and King (1965) were evaluated in a manner similar to that of Sverisson and others $(1980)$ to yield $R_{0} \approx$ $748.8 \Omega$. A satisfactory compromise between efficiency and impulse response was attained using $R_{0}=300 \Omega$, and the resistive loading was approximated by inserting fixed resistors of values found using Equation (1). These antennas have $5 \mathrm{~m}$ dipole arms that are built from $1 \mathrm{~m}$ lengths of 18 AWG copper wire with loading resistors at their midpoints. Each length is installed in a section of plastic pipe so that the antennas are rugged, collapsible, and can have loading resistors or length varied conveniently in the field. It should also be noted that, to minimize difficulties caused by transmission-line effects, the antenna should be connected directly to the current-pulse generator. Co-axial cable no longer than one-quarter of the minimum wavelength has been used successfully with our antennas.

The emitted frequency depends on the length of the antenna and the media in its vicinity. For a $5 \mathrm{~m}$ antenna half-length, our field results show that the energy radiated into the ice has a power spectrum peaking at $8.4 \mathrm{MHz}$, approximately consistent with that predicted for a simple dipole imbedded in ice (Jones, unpublished).

The actual wavelet emitted when the antenna is excited by the input voltage step is difficult to estimate analytically because the current distribution on an antenna resting on an air/ice interface is not accurately known. However, the situation can be considered qualitatively. Our damped antenna is modelled as a resistor in series with a capacitor (Wu and King, 1965). A step voltage applied to the driving point will result in a "one-lobed" current pulse; that is, current will be in one direction and will first rise and then fall. The vector potential in the far field of an antenna is proportional to the first-time derivative of the current in the antenna. By the reciprocity theorem of antennas (e.g. Jordan and Balmain, 1968 , p. 345), the voltage on a receiving antenna is related in a similar way to the far-field vector potential, so the wave form recorded by the receiver will emulate the second-time derivative of the original current pulse. Therefore, if the pulse generator can impress a step voltage on to the antenna, the best possible zero-phase echo will be a three-lobed wave form.

\section{RECEIVING UNIT}

A principal criterion for our radar receiver system was to obtain direct digital records. No commercial equipment could be found with the required degree of portability, so we designed and built the components ourselves. The present design includes a receiver amplifier, a sampling time-base (STB), an analogue-to-digital converter (ADC), a cassette tape for data storage, and a controlling computer.

The receiver amplifier is a general purpose, wide-band discrete transistor amplifier featuring fixed gain and variable front-end attenuation. The STB is a simplified version of one used in an airborne data-acquisition system described by Narod and Clarke (1983). For system control, we have developed a single-board microcomputer based on the GE/RCA COSMAC microprocessor series. Design criteria were low-temperature operation, compact packaging, a mechanically simple user interface, and flexibility in interfacing to peripherals. In all the components, many of the design choices were motivated by energy conservation. More detailed descriptions of the receiver-system components follow.

\section{Receiver}

The input stage of the receiver consists of a $0-40 \mathrm{~dB}$ variable attenuator which feeds the signal into a general purpose, five-transistor amplifier. The amplifier band width is at least $46 \mathrm{MHz}$, its voltage gain is 150 , and its input impedance is $3600 \Omega$. This is very large when compared with an ideal matched load for an undamped dipole antenna $(75 \Omega)$ or, as for our design, about $300 \Omega$. However, many investigators have found that virtually any load functions well with a receiver antenna (e.g. Watts and England (1976) successfully used a $1 \mathrm{M} \Omega$ impedance oscilloscope as a 
receiver). We selected a value of $3600 \Omega$ as a convenient input impedance for a high-speed, low-power transistor pre-amplifier

\section{Sampler}

Our goal for depth resolution was $1 \mathrm{~m}$. Using a velocity in ice of $170 \mathrm{~m} / \mu \mathrm{s}$ yields a maximum sampling interval of $11.8 \mathrm{~ns}$. We have achieved a $10 \mathrm{~ns}$ sample interval (100 $\mathrm{MHz}$ equivalent sample rate), without the high cost, large power requirements, and lower resolution of real-time digitizers, by using a sampling-time base similar to one described by Narod and Clarke (1983).

This sampling rate and our record size of 1024 points define a maximum observable two-way travel time of $10.24 \mu \mathrm{s}$. Because, in effect, we begin data acquisition before the transmitter is triggered, our recording interval is limited in practice to about $9 \mu \mathrm{s}$. Thus, our data-acquisition system is presently configured to observe a maximum depth of about $760 \mathrm{~m}$.

\section{Controller}

As noted, our instrument is controlled by a microcomputer. This computer consists of a CDP1802 central processing unit, 4 kbytes of read-only memory (containing the program), 1.25 kbytes of random-access memory (used as a data buffer), and input/output ports for communication with peripheral hardware. These peripherals include: (i) a 16-key keypad with which the user enters commands and record identifiers, (ii) an eight-digit LED display to inform the user of the system status, (iii) a digital cassette tape recorder for permanent data storage, (iv) an RS-232C serial interface for transferring data to other devices, (v) digital-to-analogue converters for displaying a record on a small, low-power oscilloscope (the Tektronix model 211 has been used for all recent field work), and (vi) the sampling time-base receiver discussed previously. The entire system is under sof tware control with the various functions initiated by the operator as instructions on the keypad. These can be classified as data collection, display, record identification, storage, and retrieval tasks, or as diagnostic functions.

Data are temporarily saved for viewing in the buffer memory, and then stored permanently on digital cassette tapes. Each record is saved as a separate file comprising 1024 eight-bit samples, and identification information entered by the operator. $25 \%$ redundant data storage, in the form of parity and cyclic redundancy characters, are included in the records and are available for error detection and correction. A maximum of 50 such records can be stored on standard digital data cassettes. The digital recorder/player and its control card are supplied by Memodyne Corporation (the model 333 "minicorder").

Data are recovered by reading records from the cassette, either individually by file number or sequentially as a complete tape, and then released to another computing system through the serial interface. Processing that has been implemented so far includes: plotting, smoothing, signal differentiation, and time-dependent amplitude variation (Prager, unpublished), as well as trace shifting or truncation, alignment across a section, spectral analysis, principalcomponent decomposition of multi-trace sections, and amplitude processing (Jones, unpublished).

\section{FIELD RESULTS}

Twenty data sets, totalling roughly 1000 individual soundings, were collected in the 1986 and 1987 summer field seasons. On safe terrain, the entire procedure of moving to a new location, then collecting, inspecting, and storing a sounding record can be accomplished by an unassisted operator in $10 \mathrm{~min}$. Field deployment and operating procedures that result in optimal system performance have been detailed in the literature (see, for example, Watts and Wright, 1981; Jezek and Thompson, 1982; Cumming and others, 1984; Jacobel and Raymond, 1984; Walford and others, 1986). In agreement with most reports, our system performance is greatest when the two antennas are oriented along two parallel lines separated by approximately one wavelength. In addition, unambiguous results can be ensured only after considering certain local features of the field site. For example we have received echoes from nearby crevasses and buried morainal material. Also, wires on the glacier surface associated with independent experiments have been observed to ring sympathetically if they are placed parallel to and within roughly one-quarter wavelength of our antennas.

Figure 4 shows sounding results from a small unnamed temperate valley glacier near Kaskawulsh Glacier, Yukon Territory, Canada. The characteristic "bow-tie" pattern
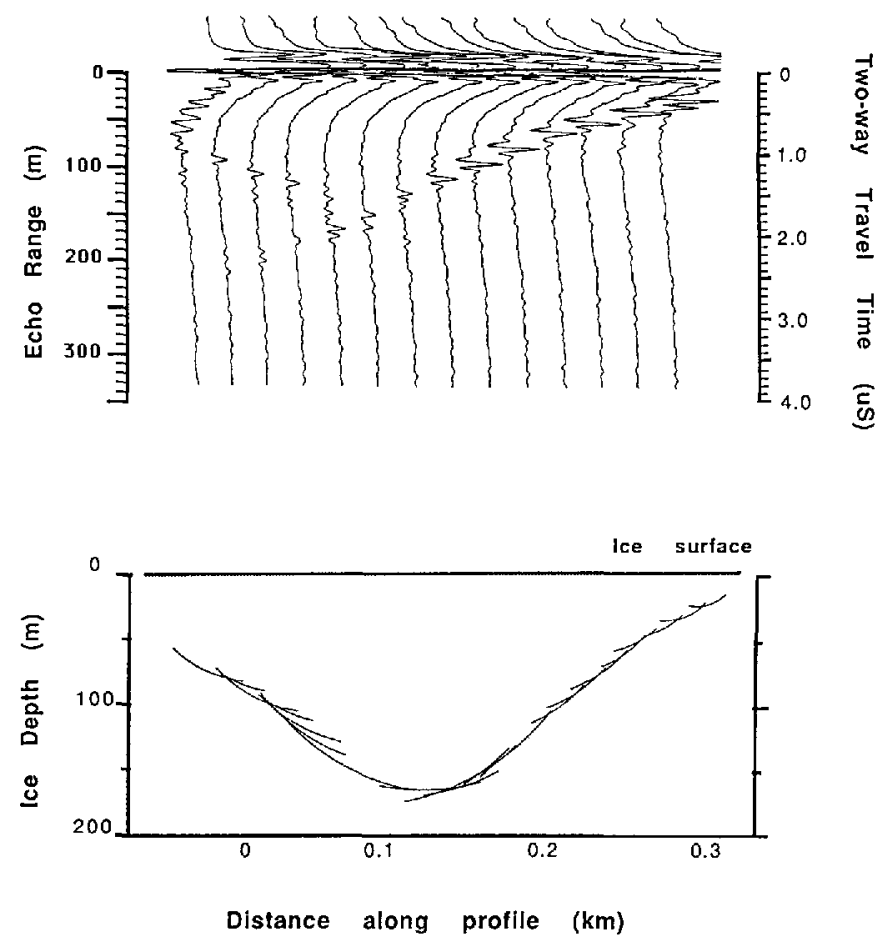

Fig. 4. Profile across a small unnamed glacier near Kaskawulsh Glacier in Yukon Territory, Canada. (a) Records were taken at $25 \mathrm{~m}$ intervals, using a transmilter and receiver spacing of $25 \mathrm{~m}$. Antennas were oriented parallel to each other and parallel to the valley walls. Data were smoothed with a three-point moving average filter before plotting (equivalent to a low-pass filter with $34 \mathrm{MHz}$ corner frequency). Note that some traces have echoes from both sides of the valley, and that echoes above the deepest points have been distorted by interference amongst closely spaced returns. Echo-range scale was calculated assuming $V_{\text {ice }}=168.2 \mathrm{~m} / \mu \mathrm{s}$. (b) Ice-depth profile constructed by drawing arcs of echo range below each record. Echoes will have returned from surfaces tangent to these arcs.

(Fig. 4a) is formed by the superposition of reflections from both walls of the subglacial channel. Records such as these are most likely to be obtained if antennas are placed parallel to the glacier margins. An arc-migrated icethickness interpretation (see Harrison, 1970) is given in Figure $4 \mathrm{~b}$.

On each trace in Figure 4a, a number of features can be recognized. After a short pre-trigger interval, the surface wave is prominent. Data were recorded using a high fixed gain, sufficient to cause severe clipping of the surface wave. Zero time was chosen as the rapidly falling edge of the surface wave. Because of phase inversion at the subglacial boundary, this feature corresponds to the rising edge of bottom reflections. For plotting, traces are aligned to the assumed zero reference and low-pass filtered to remove noise above $34 \mathrm{MHz}$; only the first $4 \mu \mathrm{s}$ of each trace are shown.

Many other experiments have been performed on temperate and sub-polar glaciers. For example, we have studied signal propagation along the ice/air interface, echoes from crevasses, and glacier-bed reflection coefficients. Temporal variations of signal character over periods of 3-5 d were compared to other, independent but concurrent experiments, and an apparently mobile subglacial feature has been observed by comparing basal echoes from the same 
geographical location recorded in subsequent years. This work is currently in progress.

\section{CONCLUSIONS}

In general, we are pleased with the performance of this prototype instrument. Improving the medium for data storage is the highest priority. The digital cassette system currently in use draws a large proportion of the receiver power and is somewhat unreliable. Our favoured solution would be to replace it with solid-state memory. This would also speed up and simplify data transfer to a field computer.

\section{ACKNOWLEDGEMENTS}

This work has been supported by the Natural Sciences and Engineering Research Council of Canada and by Northern Scientific Training Grants from the Department of Indian and Northern Affairs. We should also like to thank Dr $P$. Johnson of the Department of Geography at the University of Ottawa, Ottawa, Canada, for supporting logistics of work around Kaskawulsh Glacier. Lastly, we thank the Superintendent and staff of Kluane National Park, Yukon Territory, Canada.

\section{REFERENCES}

Brown, C.S., L.A. Rasmussen, and M.F. Meier. 1986. Bed topography inferred from airborne radio-echo sounding of Columbia Glacier, Alaska. U.S. Geol. Surv. Prof. Pap. $1258-\mathrm{G}$.

Cook, J.C. 1960. Proposed mono-cycle very-high-frequency radar for air-borne snow and ice measurement. Trans. Am. Inst. Electr. Eng., 79(1), 588-594.

Cumming, A.D.G., R.L. Ferrari, and G. Owen. 1984. Electronic design and performance of an impulse radar ice-depth sounding system used on the Vatnajökull ice-cap, Iceland. In Miller, K.J., ed. The International Karakoram Project. Vol. I, Cambridge, etc., Cambridge University Press, 111-125.

Harrison, C.H. 1970. Reconstruction of subglacial relief from radio echo sounding records. Geophysics, 35(6), $1099-1115$.
Jacobel, R.W. and S.K. Anderson. 1987. Interpretation of radio-echo returns from internal water bodies in Variegated Glacier, Alaska, U.S.A. J. Glaciol., 33(115), 319-323.

Jacobel, R.W. and C. Raymond. 1984. Radio-echo sounding studies of englacial water movement in Variegated Glacier, Alaska. J. Glaciol., 30(104), 22-29.

Jezek, K.C. and L.G. Thompson. 1982. Interpretation of monopulse ice radar soundings on two Peruvian glaciers. I.E.E.E. Trans. Geosci. Remote Sensing, GE-20(3), 243-249.

Jones, F.H.M. Unpublished. Digital impulse radar for glaciology: instrumentation, modelling and field studies. (M.Sc. thesis, University of British Columbia, 1987.)

Jordan, E.C. and K.G. Balmain. 1968. Electromagnetic waves and radiating systems. Engelwood Cliffs, NJ, Prentice-Hall Inc.

Narod, B.B. and G.K.C. Clarke. 1983, UHF radar system for airborne surveys of ice thickness. Can. J. Earth Sci., 20(7), 1073-1086.

Prager, B.T. Unpublished. Digital signal processing of UHF radio-echo sounding data from northern Ellesmere Island. (M.Sc. thesis, University of British Columbia, 1983.)

Rose, G.C. and R.S. Vickers. 1974. Calculated and experimental response of resistively loaded $\mathrm{V}$ antennas to impulsive excitation. Int. J. Electron., 37, 261-271.

Shen, L.C. and R.W.P. King. 1965. Correction to the cylindrical antenna with nonreflecting resistive loading by Wu and King. IEEE Trans. Antennas Propag., AP-13(6), 998.

Sverrisson, M., A. Jóhannesson, and H. Björnsson. 1980. Radio-echo equipment for depth sounding of temperate glaciers. J. Glaciol., 25(93), 477-486.

Walford, M.E.R., M.I. Kennett, and P. Holmlund. 1986. Interpretation of radio echoes from Storglaciären, northern Sweden. J. Glaciol., 32(110), 39-49.

Watts, R.D. and A.W. England. 1976. Radio-echo sounding of temperate glaciers: ice properties and sounder design criteria. $J$. Glaciol., $17(75), 39-48$.

Watts, R.D. and D.L. Wright. 1981. Systems for measuring thickness of temperate and polar ice from the ground or from the air. J. Glaciol., 27(97), 459-469.

Wu, T.T. and R.W.P. King. 1965. The cylindrical antenna with non-reflecting resistive loading. IEEE Trans. Antennas Propag., AP-13(3), 369-373. 